\title{
Making theory useful for understanding high-impact behavior. A response to van Valkengoed et al. (2021)
}

\author{
2021 in press, Journal of Environmental Psychology \\ Pre-print DOI 10.31234/osf.io/f2n3j
}

\author{
Florian Lange \\ KU Leuven \\ Kristian S. Nielsen \\ University of Cambridge \\ Viktoria Cologna \\ ETH Zürich \\ Cameron Brick \\ University of Amsterdam \\ Paul C. Stern
}

Social and Environmental Research Institute

\begin{abstract}
Please address correspondence concerning this article to:
Kristian Steensen Nielsen

Department of Zoology

University of Cambridge

Pembroke Street

CB2 3QZ Cambridge

United Kingdom

ksn27@cam.ac.uk
\end{abstract}

Word count: 1,068 .

\section{Declaration of conflicts of interest}

The authors report no conflicts of interest. 
In response to our article on impact-focused environmental psychology (Nielsen et al., 2021), van Valkengoed and colleagues (2021) stress the importance of theory for behavior change research. We appreciate this opportunity to clarify that we completely agree. Theory is critically needed in our collective search for actionable and generalizable knowledge on how to change environmentally significant behaviors. Yet, there are different strategies to generate behavior change knowledge and theory serves different functions within these strategies.

A first strategy may start from a psychological framework (e.g., the Theory of Planned Behavior) and aim to generate behavior change knowledge through a deductive approach to theory testing. Researchers may hypothesize that psychological constructs (e.g., environmental attitudes) are important determinants of environmentally significant behavior and then conduct an empirical test. Such empirical tests are likely to be conducted in research settings that facilitate analyzing behaviors as a function of their hypothesized determinants. Unfortunately, many behaviors high in environmental impact do not meet this criterion (e.g., because they occur infrequently or are difficult to observe). Therefore, theory testers probably would not run an intervention to change environmental attitudes and then study only rare behaviors like housing decisions or solar panel purchases. Researchers might try to address this problem by adding theoretical assumptions (e.g., that environmentally significant behaviors form a behavioral class of common psychological origin), which, if met, would allow generalizing insights from more accessible low-impact behaviors, self-reports, or hypothetical scenarios to high-impact behaviors.

Unfortunately, as argued in our article, such assumptions are rarely warranted. In addition, the psychological theories referred to by van Valkengoed and colleagues may not provide researchers (and practitioners) with sufficient behavior change knowledge. For example, 
knowing that individual differences in environmental attitude or self-efficacy correlate with an environmentally significant behavior does not reveal how to change the predictor, inspire novel interventions, or reveal causal mechanisms within the individual (e.g., Borsboom et al., 2009; Nielsen, 2017; Sniehotta et al., 2014). Observing that people are more likely to engage in behaviors that they find good or easy does not add much value to partners outside psychology nor advance behavioral explanation. In other words, theories like the ones that are often used to predict environmentally significant (and insignificant) behaviors are sometimes not of the practical kind that Lewin had in mind. This problem is not specific to environmental psychology but rather reflective of fundamental difficulties in developing good psychological theories (Eronen \& Bringmann, 2021).

In contrast, behavior change knowledge could also be acquired following an inductive approach to theory development. Instead of starting with a framework like the Theory of Planned Behavior, researchers could begin with a systematic description and analysis of the behavior of interest, characterizing the behavior and the context in which it occurs, the events that precede and follow the behavior, and the people who tend to engage in it. They might observe relationships (e.g., that the frequency of the behavior varies with the number of people within visual range) and then conduct experiments in which they systematically vary such contextual characteristics to see if the behavior varies accordingly. Researchers might then repeat this process to see if the observed regularity can also be found in other contexts and with other behaviors. This may lead to the discovery of general principles of behavioral regulation, which can be combined into a system of knowledge (i.e., an integrative theory). Constructs from existing theories can be useful under this inductive approach, but it does not bake in the assumptions that all environmentally significant behaviors have similar antecedents, that 
psychological predictors are the most important factors, or that what is learned about low-impact behaviors applies to high-impact behaviors. This inductive approach is related to recent proposals of phenomenon-driven research, which prioritize gathering robust evidence for empirical phenomena that can then constrain the space of possible theories (Eronen \& Bringmann, 2021).

We argue that such an inductive approach may help to address blind spots in research on environmentally significant behavior (e.g., knowledge about changeable determinants of highimpact behaviors). As noted by van Valkengoed and colleagues (2021), psychological theories are often used "to select psychological and contextual factors that are likely to influence the behavior." Such selection can make sense but beginning deductively from existing theory may hinder the search for the most important determinants of the most impactful behaviors. If, for example, contextual factors are selected for analysis based on their assumed relation to the constructs of psychological theories, important determinants may be overlooked. In contrast, these determinants can be identified when following an inductive, impact-focused research agenda that is less constrained by dominant psychological theories (Nielsen et al., 2021). Eventually, this approach may or may not reveal that concepts like environmental attitude or self-efficacy are useful for explaining housing decisions or solar panel purchases. Within an impact-focused research agenda, this is an empirical question rather than an a priori assumption guiding the selection of hypothesized determinants.

We hope that these clarifications help to illustrate the alternative role of theory within an impact-focused approach to studying environmentally significant behavior. Theory can be the starting point for research on environmentally significant behavior, steering the selection of behaviors, measures, and manipulations towards deductive theory tests. However, priority can 
also be given to studying high-impact behaviors, and inductive theories can then act as systems of knowledge that describe and integrate regularities to arrive at ever more general principles. In both these roles, theory supports the generation and organization of behavior change knowledge. However, we suggest that an inductive impact-focused approach is better suited to ensure that the most impactful behaviors and their most important determinants are adequately studied.

We agree with van Valkengoed and colleagues that high-impact behavior can also be studied within the predominant deductive approach. They listed several examples of studies that used high-impact behaviors to test predictions of psychological theories. We recognize and value the contributions of these studies. When theories can be tested using high-impact behaviors and when they make clear predictions on how to manipulate the determinants of behavior, deductive work can generate actionable knowledge on how to change high-impact behaviors. Yet this is often rendered difficult by the key assumptions (i.e., environmentally significant behaviors are caused by psychological constructs) and aims (i.e., testing psychological theory) of the deductive approach. We suggest that the proposed inductive, impact-focused approach can circumvent this difficulty, help integrate theory between types of behaviors, and enrich both the theoretical landscape and practical usefulness of environmental psychology. 


\section{References}

Borsboom, D., Kievit, R. A., Cervone, D., \& Hood, S. B. (2009). The two disciplines of scientific psychology, or: The disunity of psychology as a working hypothesis. In J. Valsiner, P. Molenaar, M. Lyra, \& N. Chaudhary (Eds.), Dynamic process methodology in the social and developmental sciences (pp. 67-97). Springer, New York, NY.

Eronen, M. I., \& Bringmann, L. F. (2021). The theory crisis in psychology: How to move forward. Perspectives on Psychological Science. https://doi.org/10.1177/1745691620970586

Nielsen, K. S. (2017). From prediction to process: A self-regulation account of environmental behavior change. Journal of Environmental Psychology, 51, 189-198. https://doi.org/10.1016/j.jenvp.2017.04.002

Nielsen, K. S., Cologna, V., Lange, F., Brick, C., \& Stern, P. (2021). The case for impactfocused environmental psychology. Journal of Environmental Psychology, 101559. https://doi.org/10.1016/j.jenvp.2021.101559

Sniehotta, F. F., Presseau, J., \& Araújo-Soares, V. (2014). Time to retire the theory of planned behaviour. Health Psychology Review, 8, 1-7. https://doi.org/10.1080/17437199.2013.869710

van Valkengoed, A. M., Steg, L., Perlaviciute, G., Schultz, P. W., Brosch, T., Gatersleben, B., Nordlund, A., Pahl, S. \& Whitmarsh, L. (2021). Theory enhances impact. Reply to: 'The case for impact-focused environmental psychology’. Journal of Environmental Psychology. https://doi.org/10.1016/j.jenvp.2021.101597 